\title{
Genetic insights into the regulatory pathways for continuous flowering in a unique orchid Arundina graminifolia
}

\author{
Sagheer Ahmad, Chuqiao Lu, Jie Gao, Rui Ren, Yonglu Wei, Jieqiu Wu, Jianpeng Jin, Chuanyuan Zheng, \\ Genfa Zhu* and Fengxi Yang*
}

\begin{abstract}
Background: Manipulation of flowering time and frequency of blooming is key to enhancing the ornamental value of orchids. Arundina graminifolia is a unique orchid that flowers year round, although the molecular basis of this flowering pattern remains poorly understood.

Results: We compared the A. graminifolia transcriptome across tissue types and floral developmental stages to elucidate important genetic regulators of flowering and hormones. Clustering analyses identified modules specific to floral transition and floral morphogenesis, providing a set of candidate regulators for the floral initiation and timing. Among candidate floral homeotic genes, the expression of two FT genes was positively correlated with flower development. Assessment of the endogenous hormone levels and qRT-PCR analysis of 32 pathway-responsive genes supported a role for the regulatory networks in floral bud control in A. graminifolia. Moreover, WGCNA showed that flowering control can be delineated by modules of coexpressed genes; especially, MEgreen presented group of genes specific to flowering.
\end{abstract}

Conclusions: Candidate gene selection coupled with hormonal regulators brings a robust source to understand the intricate molecular regulation of flowering in precious orchids.

Keywords: Arundina graminifolia, Flowering, Hormone signaling, WGCNA, Transcriptome

\section{Background}

Flowering plants are the dominant component of terrestrial landscape and play a key role in human life. The time of flowering is crucial for the species to adapt in their environment as their life cycle runs depending on the fine combination of intrinsic and extrinsic stimuli. From the perspective of horticultural importance, the flower timing often affects the quality of floriculture crops. Stability of flowering time is the key goal for breeding endeavors

\footnotetext{
*Correspondence: zhugenfa@gdaas.cn; yangfengxi@gdaas.cn Guangdong Key Laboratory of Ornamental Plant Germplasm Innovation and Utilization, Environmental Horticulture Research Institute, Guangdong Academy of Agricultural Sciences, Guangzhou 510640, People's Republic of China
}

because it guarantees the reliable production of crops. In recent decades, mining of genes regulating flowering pathways has been the major focus to induce breeding novelty in floriculture crops. Information from different pathways is synthesized through floral integrator genes, whose expression instigates the transition of apical meristem from vegetative to floral phase [1].

Environmental variations, such as changes in light, temperature, and hormone levels, control flower quality and flowering time [2]. Flowering time in Arabidopsis depends on five genetic pathways: photoperiod, aging, gibberellin, vernalization, and autonomous pathways [3]. These pathways are integrated by floral integrators, including SUPPRESSOR OF OVEREXPRESSION OF CO 
1 (SOC1), FLOWERING LOCUS D (FD), and FLOWERING LOCUS T (FT) [2-4]. These integrators trigger the floral morphogenesis program by transmitting the floral induction signals to the floral meristem identity genes, including APETALA1 (AP1) and LEAFY (LFY). Then, floral organ development continues under the control of MADS-box genes and their co-regulators [4].

Orchids contain several MADS-box genes that regulate flowering and flower development. Based on their expression patterns and putative roles in floral organs, advanced models have been proposed for flower development in orchids [5, 6]. Recently, an increasing number of flowering genes have been functionally characterized in various orchid species, including homologs of $F T$ and the gene that encodes its interacting protein, $F D$, in Phalaenopsis aphrodite, Oncidium Gower Ramsey, and Dendrobium nobile; CONSTANS-like genes and LEAFY in Phalaenopsis aphrodite; and genes for co-regulated transcription factors such as CINCINNATA-like (TCPlike) and SQUAMOSA promoter binding-like genes (SPLlike) [7-12].

Some of these homologs exhibit conserved functions in orchids. PaFT1 (Phalaenopsis aphrodite FLOWERING LOCUS T1) is upregulated at low temperatures and induces early flowering in rice (Oryza sativa) and Arabidopsis [13]. PaFT1-interacting protein (PaFD) partially complements the late-flowering phenotype of the Arabidopsis mutant fd-3 [13]. OnFT (Oncidium Gower Ramsey FLOWERING LOCUS T) and TFL1 (TERMINAL FLOWER 1) encode the orchid homologs of the floral activator FT and the repressor TFL1, respectively [7]. The balance between these homologous proteins (FT and TFL1) controls the determinate and indeterminate growth in plants and also modulates plant architecture, thereby regulating the vegetative and reproductive organ pattern from apical meristem $[14,15]$. Dendrobium nobile MOTHER OF FT (DnMFT) and FLOWERING LOCUS T (DnFT) show opposite expressions against change in temperature. Together, these studies inform our understanding of the relationships between flower development and floral regulatory genes in orchids [16].

Phytohormones play important roles in orchid flowering [17]. Auxin acts as a morphogen [18-22] and provides cues for tissue specification in a concentration-dependent manner [23]. Application of a synthetic cytokinin, 6-benzylaminopurine (BA), promoted flowering in Phalaenopsis and Dendrobium orchids, but auxin counteracted this effect. The effect of BA on flowering is enhanced when applied in combination with gibberellic acid $\left(\mathrm{GA}_{3}\right)$ [24]. GAs regulate important developmental processes like flowering time $[25,26]$ and stem elongation [27-29]. Abscisic acid (ABA) is a pivotal factor that regulates bud break and flowering time [30,31].
Strigolactones also play important roles through their cross-talk with other hormones, such as auxin, cytokinin, GA, and ethylene [32-34]. Despite their potential for wide use, the molecular underpinnings of the effects of phytohormones on orchid flowering remain unclear.

Orchidaceae is one of the largest angiosperm families and contains ornamental orchids [35, 36]. Because of their horticultural importance, more than 100,000 orchid species have been cultivated throughout the world. Most commercially popular orchid cultivars, such as Phalaenopsis, Dendrobium, and Cymbidium, bloom in specific seasons, under low temperature of about $5-10^{\circ} \mathrm{C}$ [37]. Unlike these orchids, Arundina graminifolia blooms year round, peaking between September and January. It is commonly known as the "Bamboo Orchid" and is found in tropical and sub-tropical regions in Asia [38-41]. It grows on grassy slopes, under shrubs, along ravines, or in forest areas, at altitudes between 400 and $2800 \mathrm{~m}$, showing a strong adaptability and a high rate of flowering and fruiting throughout the year [41]. Due to its beautiful flowers and extended flowering time, A. graminifolia is widely grown in Singapore, Malaysia, among other places, as a landscape and potted plant. A. graminifolia is used as a medicinal plant in China because of the presence of flavonoids, stilbenoid, and phenols in its extracts, which exhibit antioxidant, anti-virus, antitumor, and other medicinal properties [42]. Researchers have so far focused on the identification of the chemical components, pharmacological activities, and bioactive substances in A. graminifolia. However, the underlying molecular regulation is not discussed.

Here, we report the first de novo transcriptomic analysis of A. graminifolia. We compared the transcriptomic profiles at different stages of flower development and those of vegetative tissues. We elucidated the transcriptome dynamics and the gene regulatory networks associated with flower development. We identified highly expressed gene modules, unique flower-specific markers, circadian clock integrators, and hormonal regulators in different flower development stages, and in mature flowers, capsules, leaves, and roots. Our study, thus, provides valuable insights into the molecular regulatory network that regulates flowering in A. graminifolia.

\section{Results}

Perpetual flowering and flower ontogeny of A. graminifolia A. graminifolia achieves reproductive maturity 6 months after transplantation from a culture medium. This is much shorter than the two- to three-year long juvenile phase of other orchids, such as Phalaenopsis, Oncidium, Dendrobium, and Cymbidium. A. graminifolia blooms all year round, with the most vigorous flowering from September to January (Supplementary Fig. 1). 
Its inflorescence is a raceme with an average of 6.1 flowers per plant. The average life span of a single flower is 32.3 days. The ornamental period of the whole inflorescence may span up to 5 months (Table 1 ).

We observed the morphogenesis and surface structure of the floral organs under a scanning electron microscope (Fig. 1). Flower development takes place in six stages (stages $0-5$ ). In stage 0 , the inflorescence meristem (IM) is formed and has a flattened and oval primordium (Fig. 1a). In stage 1, the IM displays a floral structure on the edge of the shoot, and the floral primordia divide into sepal, petal, labellum, and column primordia. Even though the process is similar to that of other orchid species, the pace of development is much faster, with a completion time of 2-3 days in A. graminifolia. The floral organs are undifferentiated at this stage, and the base is connected (Fig. 1b,c). In stage 2, the floral organs differentiate continuously and establish the zygomorphy typical of orchid flowers (Fig. 1d). It takes about 10 days to grow into stage 3 . In this stage, the floral apex resembles an inverted triangle (Fig. 1e): the outer sepals overlap with the inner petals (Fig. If and g), and the labellum develops a crooked to undulated margin (Fig. 1h). Stage 4 commences after 1 week, wherein the gynostemium elongates and the labellum acquires its coloring (Fig. 1i). Then the pollinia mature and the flower opens within 3 days (stage 5, Fig. $1 \mathrm{~m}$-p). The mature flower has a finestructured column in the center (Fig. 1o), with four pollinia on a semi-circular viscidium (Fig. 1p).

\section{Transcriptome sequencing and functional annotation}

We produced a total of 71.2 billion high-quality reads from. Each sample produced 10.8-12.8 Gb of data and an average of $\sim 7.8$ billion reads (Supplementary Table 1 ). We filtered these into 94,317 transcripts and 25,353 unigenes (Supplementary Table 2), with mean lengths of $1291 \mathrm{bp}$ and $1198 \mathrm{bp}$, respectively. We annotated them using Gene Ontology (GO), Kyoto Encyclopedia of Genes and Genomes (KEGG), database of protein families (Pfam), SwissProt, and evolutionary genealogy of genes: Non-supervised Orthologous Groups (eggNOG) and non-redundant (NR) protein databases (Table 2). We identified a total of 17,171 (67.73\%) unigenes with GO annotations for biological processes, molecular functions, or cellular components. We annotated 9234 genes into KEGG categories. We obtained the maximum number of annotations using eggNOG, where 18,854 (74.37\%) reads were assigned to different categories, followed by NR database in which $72.29 \%$ of the reads were annotated to non-redundant proteins.

Signal transduction, posttranslational modification, transcription, carbohydrate transport and metabolism, intracellular trafficking and amino acid transport, and metabolism were the major functional categories in the transcriptomic data annotated by eggNOG (Supplementary Fig. 2a). In GO annotations, a significant number of genes were annotated to transcriptional regulation, transcription, and protein phosphorylation. Most of the genes were localized to nucleus, cytoplasm, membranes, and chloroplast. Protein binding was the most prominent molecular function across all the annotated genes (Supplementary Fig. 2b). Most KEGG-annotated genes were related to translation, carbohydrate metabolism, and lipid and energy metabolism (Supplementary Fig. 2c).

A. graminifolia shared 7820 unigenes with orchids, such as Phalaenopsis equestris, Dendrobium officinale, and Apostasia shenzhenica. In pair-wise comparisons, $A$. graminifolia shared 8778, 9925, and 10,012 genes with $P$. equestris, D. officinale, and $A$. shenzhenica, respectively (Supplementary Fig. 2d). Only 64 genes were unique to A. graminifolia, compared with 615 in P. equestris, 1132 in D. officinale, and 615 in A. shenzhenica. The GO analysis resulted in 136 annotated terms for genes unique to A. graminifolia; those involved in biological process (80), metabolic process (34), and response to stimulus (27) were relatively over-represented. However, only 17 terms were annotated to cellular components, and 1 to the molecular function category.

\section{Identification of Transcription Factor (TF) families}

Our data contained 687 TFs belonging to 35 different families (Supplementary Fig. 4). Famous TF families were

Table 1 Morphology of reproductive growth of A. graminifolia

\begin{tabular}{|c|c|c|c|c|c|c|c|}
\hline \multirow{2}{*}{$\begin{array}{l}\text { Time after } \\
\text { first blooming } \\
\text { (month) }\end{array}$} & \multirow{2}{*}{$\begin{array}{l}\text { Number of } \\
\text { leaves }\end{array}$} & \multirow{2}{*}{$\begin{array}{l}\text { Plant height } \\
\text { (cm) }\end{array}$} & \multirow{2}{*}{$\begin{array}{l}\text { Stem diameter } \\
(\mathrm{mm})\end{array}$} & \multicolumn{4}{|l|}{ Pedicel } \\
\hline & & & & Section number & Diameter (mm) & Length $(\mathrm{cm})$ & Total length $(\mathrm{cm})$ \\
\hline 1 & $17.05 \pm 0.64^{\mathrm{a}}$ & $34.05 \pm 1.51^{\mathrm{a}}$ & $4.23 \pm 0.11^{\mathrm{a}}$ & $2.00 \pm 0.00^{\mathrm{a}}$ & $0.85 \pm 0.03^{\mathrm{a}}$ & $1.62 \pm 0.20^{b}$ & $3.23 \pm 0.40^{b}$ \\
\hline 2 & $15.85 \pm 0.55^{b}$ & $34.48 \pm 1.48^{b}$ & $3.83 \pm 3.76^{b}$ & $3.05 \pm 0.17^{b}$ & $1.69 \pm 0.04^{b}$ & $2.82 \pm 0.16^{c}$ & $6.90 \pm 0.70^{\mathrm{a}}$ \\
\hline 3 & $15.45 \pm 0.60^{b}$ & $35.57 \pm 1.39^{c}$ & $3.59 \pm 0.08^{c}$ & $4.50 \pm 0.48^{c}$ & $1.64 \pm 0.04^{b}$ & $1.62 \pm 0.08^{b}$ & $12.61 \pm 1.17^{c}$ \\
\hline 4 & $14.13 \pm 0.60^{c}$ & $36.03 \pm 1.47^{d}$ & $3.13 \pm 0.08^{d}$ & $5.73 \pm 0.40^{d}$ & $1.40 \pm 0.05^{c}$ & $1.39 \pm 0.09^{\mathrm{a}}$ & $15.00 \pm 1.29^{d}$ \\
\hline 5 & $12.75 \pm 0.63^{d}$ & $36.21 \pm 1.48^{d}$ & $3.07 \pm 0.08^{d}$ & $6.45 \pm 0.50^{e}$ & $1.40 \pm 0.09^{c}$ & $1.38 \pm 0.18^{\mathrm{a}}$ & $15.22 \pm 1.35^{e}$ \\
\hline
\end{tabular}

The symbols $(\mathrm{a}, \mathrm{b}, \mathrm{c}, \mathrm{d}, \mathrm{e})$ show statistically significant difference at $p<0.05$ 

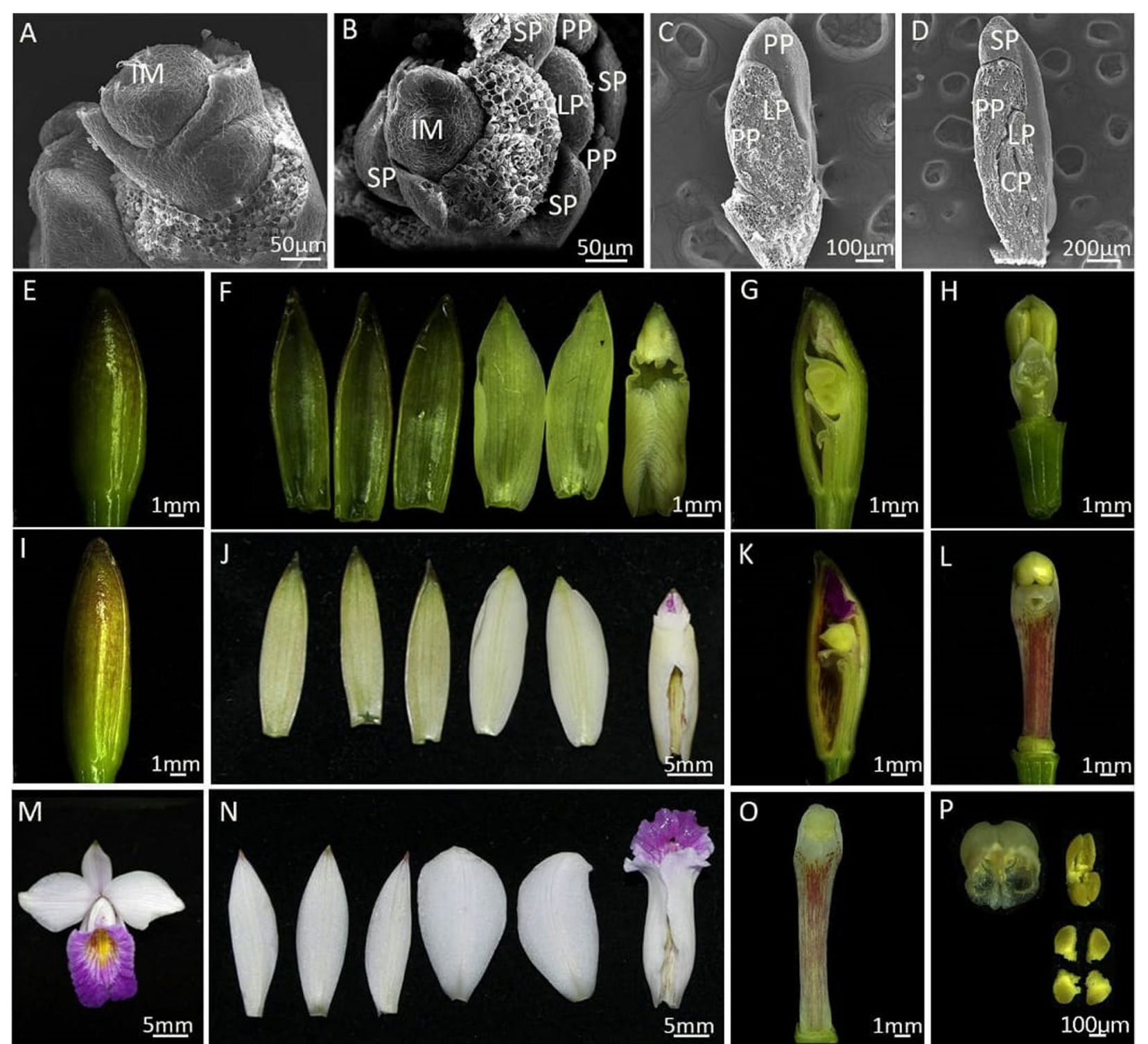

Fig. 1 Stages of flower development in Arundina graminifolia. A-D: Scanning electron micrograph (SEM) of early floral developmental stages. A: Stage 0 (IM: inflorescence meristem); B-C: Stage 1 (IM: inflorescence meristem, PP: petal primordia, SP: sepal primordia); D: Stage 2 (PP: petal primordia, SP: sepal primordia, LP: lip primordia, CP: carpel primordia); E-H: Stage 3; I-L: Stage 4; M-P: Stage 5; P: mature flower

Table 2 Annotation statistics of DEGs for different databases

\begin{tabular}{lll}
\hline DB & Num & Ratio (\%) \\
\hline All & 25,353 & 100.00 \\
GO & 17,171 & 67.73 \\
KEGG & 9234 & 36.42 \\
Pfam & 16,271 & 64.18 \\
SwissProt & 14,334 & 56.54 \\
eggNOG & 18,854 & 74.37 \\
NR & 18,327 & 72.29 \\
\hline
\end{tabular}

found in the transcriptome, including bHLH, WRKY, MYB, CYC, bZIP, TCP, and MAD. The most abundant TF family was bHLH (70 members), followed by MYB (50 members) and WRKY (45 members). The bHLHs and MYBs were expressed significantly in the root, leaf, and early FD stages. However, WRKYs were mainly expressed in the root and flower (Supplementary Fig. 3).

Tissue specific up- and downregulation modules were found for TF families (Supplementary Fig. 5). Interesting variations can be found among TFs families for stages of flower development or tissue types (Supplementary 
Fig. 4a). The most upregulated TFs (435) were found in FD1, whereas the most downregulated TFs (495) were found in FD5 (Supplementary Fig. 5b). An equal ratio of up- and downregulated TFs can be seen in the mature flower (Supplementary Fig. 4c).

\section{Differential expression of flowering-related genes}

We identified 36 floral-related DEGs (Fig. 2a). Genes encoding homologs of the floral regulators, $S E P, E L F$, $M A D S$, TCP, AGL, FT, and LHP, were expressed in FD1 (Fig. 2a). ELF works in the photoperiodic pathway (GO: 0009648) and controls rhythmic process (GO: 0048511) of organ development. TCP21 is a transcription factor that regulates the circadian rhythm (GO: 0042752).

Two isoforms of MADS6 and FT showed high expression in FD2. MADS6 is involved in the specification of floral organ identity and meristem determinacy (K03217). An isoform of COL9 was the only CONSTANS-like zinc finger protein expressed in FD3. It regulates circadian rhythms (GO: 0007623) and flowering (GO: 0048579).
AGAMOUS-like (AGL) MADS-box proteins (AGL104, AGL61, and AGL65) were expressed in FD4, involving pollen development (Fig. 2a). Moreover, COL (CONSTANS-like zinc finger protein) was also expressed in FD4 and it controls regulation of flower development (KO3539). In stage 5 of flower development, AGL11 and EFLs were upregulated. EFL3 and 4 are floral repressors that coordinate floral transition through the photoperiod pathway.

Two types of COs, two EFL types, GI, and PIF3 were expressed in mature flower (Fig. 2a). GIGANTEA (GI) is a protein that regulates the circadian clock (K12124). We observed a high level of GI expression in mature flower.

\section{Validation of AgFT genes correlated with reproductive development}

Flowering Locus T (FT) family genes function as key flowering integrators: FT promotes flowering and TFL1 inhibits flowering. To comprehensively identify the FT genes in A. graminifolia, we used the Arabidopsis and rice FT amino acid sequences to screen for homologs in our

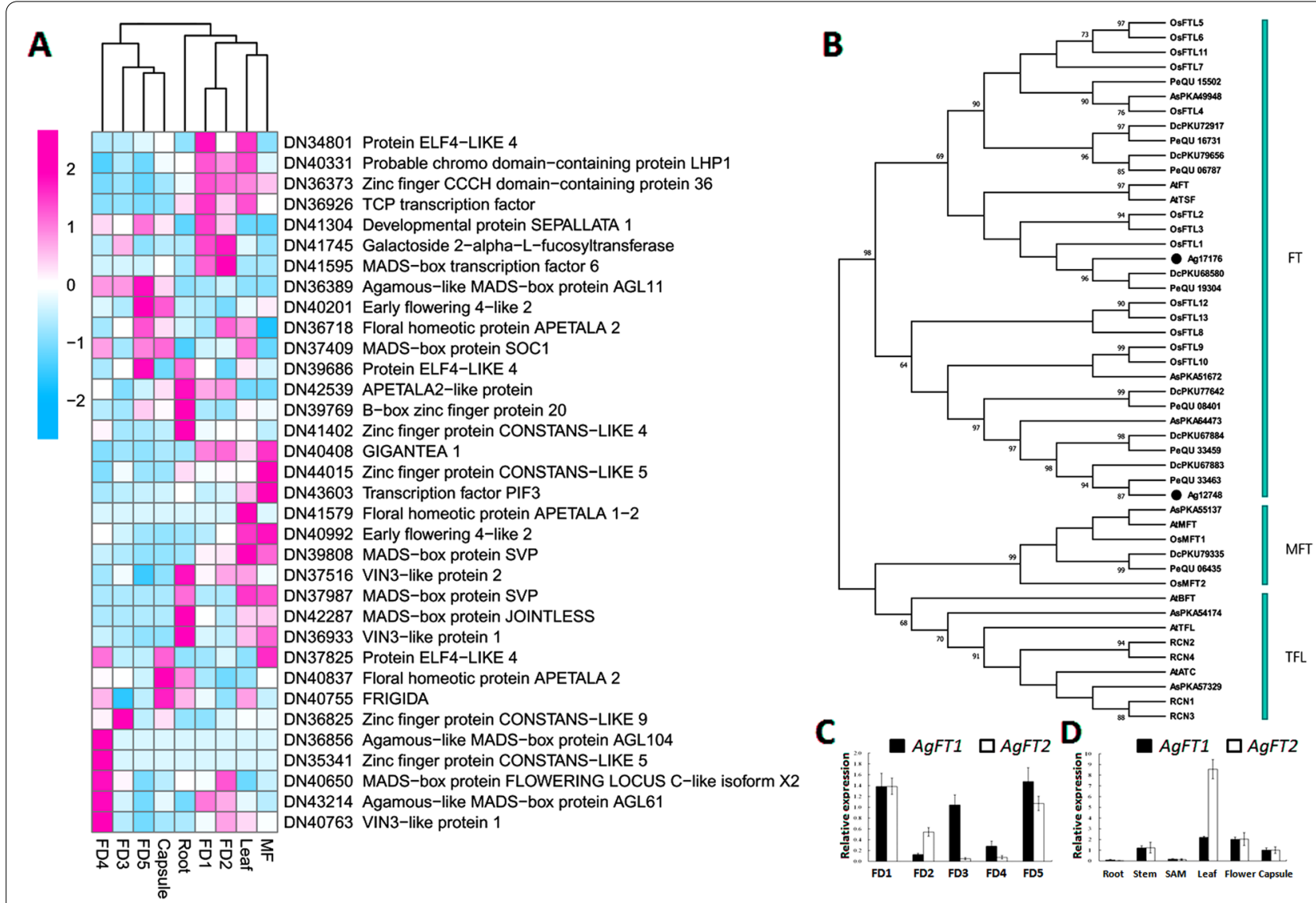

Fig. 2 DEGs relating to flowering control. A: heatmap of flowering-related DEGs; B: Phylogenetic tree of AgFT genes with FT, MFT, and TFL genes from other species; C: qRT-PCR expression of AgFT1 and AgFT2 in flower development stages; D: qRT-PCR analysis of AgFT1 and AgFT2 in tissues other than flower 
dataset using tBLASTN. FT gene number varied among species (Fig. 2b). There are 6, 7, and 8 FT-like genes in Apostasia, Phalaenopsis, and Dendrobium, respectively. However, A. graminifolia contained only 2 FT-like genes, and no TFL1-like or MFT-like genes.

$A g F T$ genes showed the lowest expression levels in root and shoot apical meristem (SAM), and high levels in leaf and flowers, followed by stem and capsule (Fig. 2d). This pattern is similar to the FT expression pattern in other species, and is associated with floral bud initiation. Both $A g F T 1$ and $A g F T 2$ were highly expressed in floral developmental stages 1 and 5, but had lower expression in stages 2-4 (Fig. 2c). Moreover, we observed a complementary expression pattern of $A g F T 1$ and $A g F T 2$ during stages 2-4: AgFT1 showed higher expression in stage 2, while $A g F T 2$ showed higher expression in stages 3 and 4.

\section{Hormonal regulation possesses a fundamental role in flower development}

We mined a number of genes involved in the biosynthesis and signalling of auxins (Fig. 3a). Notably, TAR2, a candidate gene involved in auxin biosynthesis (GO: 0009851), was expressed only in FD4. SKP2A, responsible for auxinactivated signaling (GO: 0009734), was expressed in FD2
(Fig. 3a). Auxin efflux carriers (PINs) were expressed mainly in FD4, and ARFs showed high expression in the capsule or early stages of flower development.

Cytokinins interact with auxins to control organogenesis. We observed higher expression of cytokinin-related genes in FD4 than in other stages of flower development, or in other tissue types (Fig. 3a). LOGs are involved in cytokinin biosynthesis (GO: 0009691). LOG7 was expressed in FD4. LOG1 appeared in three isoforms, in leaf, FD5, and FD1. CRF3, which acts in the cytokinin-activated signaling pathway (GO: 0009736), was expressed only in FD4. CRF5 exhibited low expression in FD4 and elevated expression in root and FD1.

We also identified genes involved in strigolactone biosynthesis and signaling (Fig. 3a). These genes were not expressed in the first three stages of flower development. The highest expression levels were in FD4 and roots. D27 is responsible for strigolactone biosynthesis (GO: 1901601) and was only expressed in leaf. CCD7, another strigolactone biosynthesis gene (GO: 1901601), showed highest expression levels (FPKM: 164.07) in FD4. $S M A X 1$, a transcriptional corepressor that confers to response to strigolactone (GO: 1902347), also displayed high expression levels in FD4 (Fig. 3a).

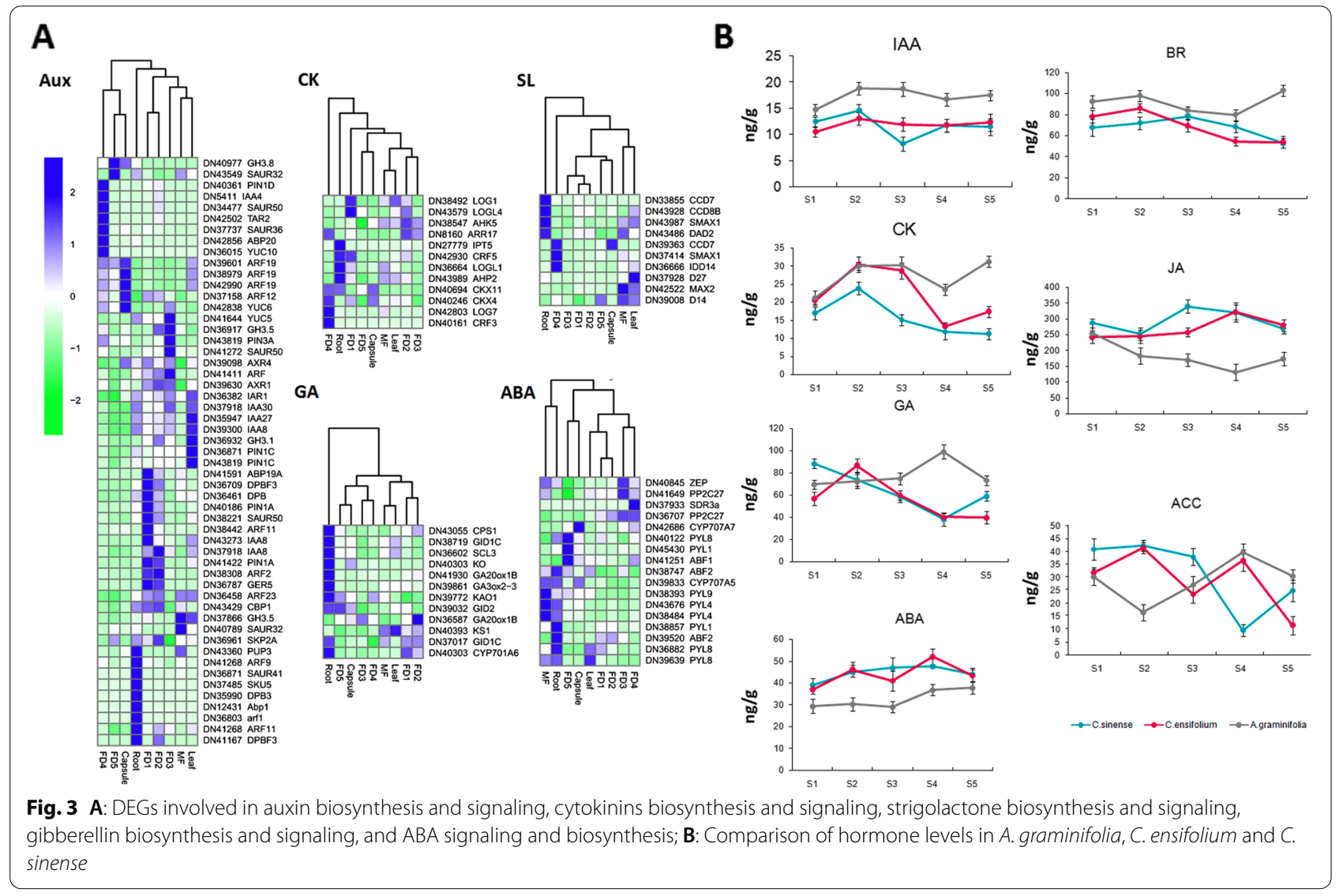


We identified 72 annotated genes related to gibberellin. A heatmap of these annotations showed that most of the genes had high expression levels in root, followed by FD1 and FD2 (Supplementary Fig. 5). Two DELLA genes (GAI, SLR-like) were repressed along flower development (Fig. 3a). Reduced expression of DELLA genes promotes floral induction and development.

Abscisic acid is a general negative regulator of plant organ development, and interacts with other hormones to control flowering. We identified 19 transcripts related to ABA biosynthesis, metabolic process, ABA-activated signaling, and ABA binding. Most of these genes showed significant expression intensities in roots (Fig. 3a).

\section{Correlation between the endogenous hormones and flower development}

To validate the effect of hormones on flower development, we investigated the levels of endogenous hormones-cytokinins (CK), gibberellic acid (GA), abscisic acid (ABA), brassinosteroid (BR), jasmonic acid (JA), 1-Aminocyclopropane-1-carboxylic acid (ACC), and indoleacetic acid (IAA)-at different growth stages (Fig. 3b). These were then compared with the corresponding hormone levels in the seasonal-flowering orchid species, Cymbidium sinense (spring) and Cymbidium ensifolium (summer).

All hormones except ACC varied significantly among these orchids. A. graminifolia had 1.3- to 2.5-fold higher levels of GA3, CK, IAA, and BR than the seasonal-flowering orchids. The levels of these hormones increased during floral bud development: FD5 buds had 13-50\% more hormones than FD1 buds. In a reversal of this pattern, FD5 buds of $C$. sinense and $C$. ensifolium had 20-40\% lower levels of these hormones than FD1 buds. A. graminifolia had considerably lower levels of JA and $\mathrm{ABA}$ - which are negative regulators of floral organ development-than C. sinense and C. ensifolium (Fig. 3b).

We validated the hormone responsive genes in the GA and ABA pathways by qRT-PCR. This analysis included five GA-related genes (GID1, GA20OX, GA3OX, GAI, and $S L R)$ and four ABA-related genes (ABF1, $A B F 2$, PYL4, and CYP707A5; Fig. 5b). GA3OX was expressed predominantly in the root, and GID1 and GA20OX were expressed to comparable levels in all plant parts. The DELLA genes (GAI and SLR) showed decreasing expression levels during bud development, corroborating the DEG analysis. $A B F 1$ had higher expression levels in FD1 than in other stages or tissues. $A B F 2$ showed high expression in FD1 and FD2. CYP707A5 was expressed mainly in roots, and its levels decreased during floral bud development. PYL4 was expressed mainly in leaf and root.

\section{Identification of coexpressed gene modules for flowering}

To better understand the gene regulatory networks during flower development, we performed WGCNA in association with flowering and tissues (Fig. 4). Six

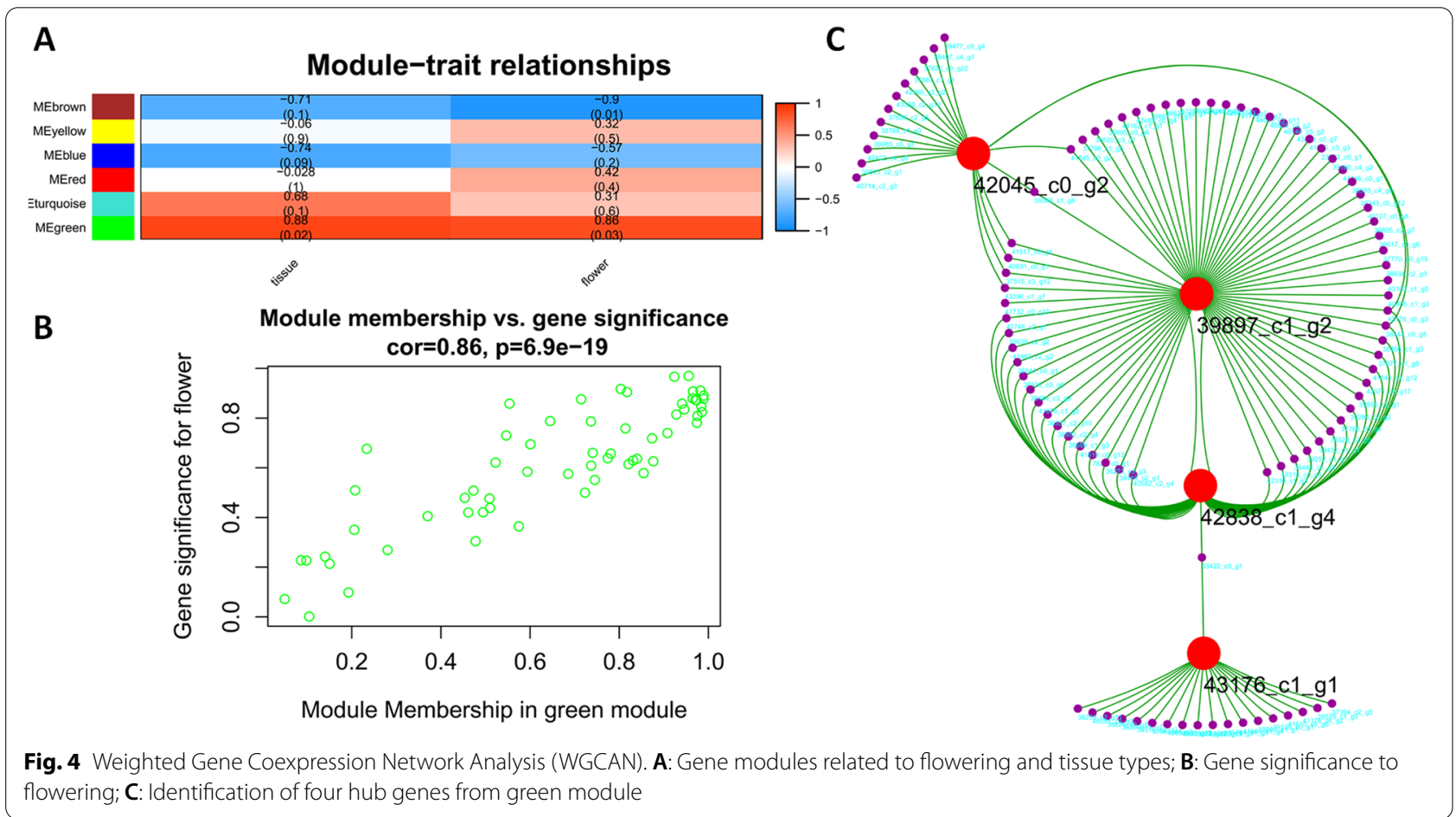




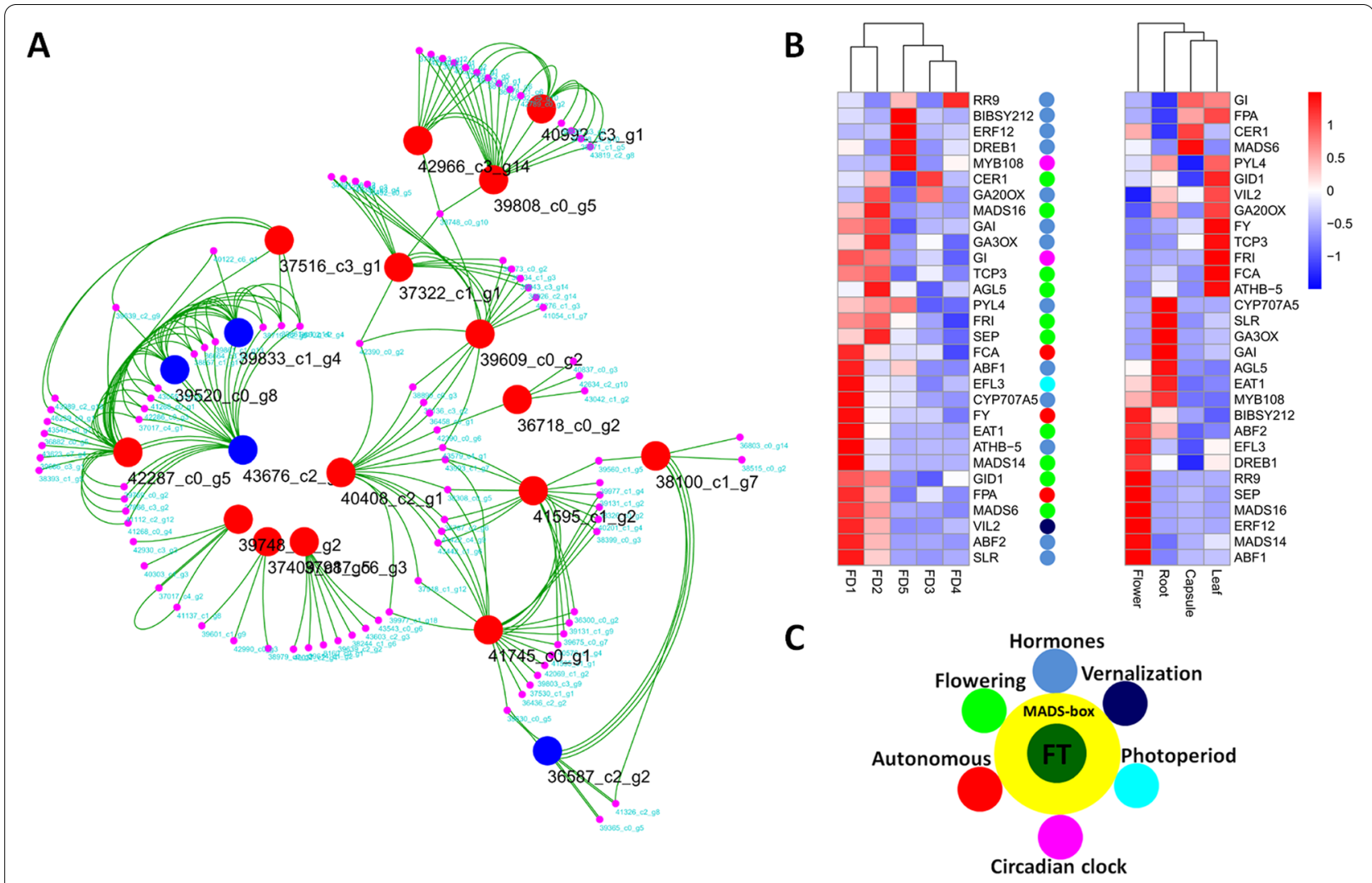

Fig. 5 Hub genes from coexpressed modules related to flowering and hormonal regulation (A) (blue circles show the key hormonal regulators and the red circles show floral regulators), qRT-PCR analyses of 30 selected genes related to multiple pathways (B) and the hypothetical model of flowering regulation by different pathways (C)

modules were observed for flowering. MEgreen was the most prominent for flowering (Fig. 4a). A cluster of genes can be seen with a high correlation value 0.86 in MEgreen module (Fig. 4b). From this module, some hub genes were identified using Cytoscape with their intra- and inter-module interactions (Fig. 4c).

The 39897_c1_g2 is annotated as DRE-binding protein DREB1. It is an ethylene-responsive transcription factor (RAP2-13) with GO annotation as ethylene-activated signaling pathway (GO: 0009873). 43176_c1_g1 is a bibenzyl synthase (BIBSY212), involving various biological processes, such as response to auxin (GO: 0009733), response to jasmonic acid (GO: 0009753), flavonoid biosynthetic process (GO: 0009813) and auxin polar transport (GO: 0009926). 42045_c0_g2 is annotated as protein ECERIFERUM (CER1) with biological process of anther development (GO: 0048653). 42838_c1_g4 is predicted to be homeobox-leucine zipper protein ATHB-5 and may involve ABA regulation, including response to ABA (GO: 0009737),
ABA-activated signaling pathway (GO: 0009738) and regulation of $\mathrm{ABA}$-activated signaling pathway.

\section{The qRT-PCR validation of selected DEGs}

All the DEGs related to flowering and hormonal regulation were sorted and coexpressed modules were generated. This identified a number of hub genes that play important roles in flowering (Fig. 5a). A total of 32 DEGs were quantified through qRT-PCR to ascertain their roles in flower development (Fig. 5b). The qRTPCR expressions of most of the DEGs were consistent with that of transcriptomic expressions. Clustering of qRT-PCR expressions showed that most of the DEGs were expressed in FD1 among the stages of flower development, representing different pathways of flowering regulation. Most of the qRT-PCR detected DEGs showed similarities to the genes identified in other orchids, such as Cymbidium sinense, Cymbidium goeringii, Dendrobium catenatum, and Phalaenopsis equestris (Supplementary Table 4). Moreover, a hypothetical 
model can be proposed based on the variety of regulatory genes found in our data (Fig. 5c).

\section{Discussion}

Arundina graminifolia is unique among orchids due to continuous flowering, but the molecular regulation of this flowering pattern remains poorly understood. We used de novo RNA-seq to study the transcriptome dynamics of flower development. Several genes were expressed during flower development stages and in various tissue types. We identified homologs of key flowering regulators, such as ELF, COL4, COL5, COL9, FT, and SOC1, in A. graminifolia. Homologs of GA20ox $1 B$, GID1C, GID2, GA3ox2, GAI, and SLR in the GA pathway, ABA pathway genes (ABF1, ABF2 and PYL4) and homologs of $F C A, F P A$, and $F Y$ in the autonomous pathway were identified in this study. Important transcription factor families were also rich in the data. Contents of seven hormones were quantified in A. graminifolia and compared with $C$. sinense and $C$. ensifolium. Therefore, our data from A. graminifolia captures genes from classical flowering pathways that have been identified in Arabidopsis [3].

Our data suggested important TFs that expressed to specific stages of flower development (Fig. 5b). MADS14 is an AP1 (APETALA1)/FUL (FRUITFUL)-like MADSbox TF that regulates floral meristem identity [43-45]. It showed the highest expression in FD1 as compared to other stages of FD (Fig. 5b). MADS6, MADS16 and SEP showed high expressions in FD1 and FD2. MYB108 along with MYB24 regulates JA-mediated stamen maturation in Arabidopsis [46]. It was highly expressed in FD5. EAT1 (EXTERNAL TAPETUM1) is a bHLH TF involving tapetal cell-fate decision [47] and showed high expression in FD1. RR9 (type-B response regulator) showed high expression in FD4 and FD5. ERF12 is a transcriptional repressor in the photoperiod pathway and regulates floral timing and floral organ identity along with AP2 (APETALA2) $[48,49]$. It was mainly expressed in FD5 (Fig. 5b).

The transcriptome of $A$. graminifolia contained two FT homologs, but no TFL1 homologs. FT genes provide important sources of flowering-time variation in different plant species or cultivars. $F T$ is regulated antagonistically by the photoperiod and vernalization pathways and plays a central role in integrating flowering [50]. Sequence comparisons clustered the FT homologs into three major clades: the FT, MFT, and TFL1 subfamilies. FT and MFT function as flowering promoters, whereas TFL1 is associated with vegetative development and maintenance of meristem indeterminacy. Seasonal orchid species contain comparable numbers of flowering-related MADS-box.
The contraction of the FT gene family members may be a unique characteristic of this species.

Bud growth is promoted by endogenous hormones such as GA3, CK, IAA, and BR, and is inhibited by ABA, JA, and ACC [51-56]. We quantified the hormonal contents in A. graminifolia compared with the typical seasonal flowering species (C. sinense and $C$. ensifolium). The levels of GA3, CK, IAA, and BR in A. graminifolia are significantly higher than those in seasonal-flowering orchids. In line with our expectations, the levels of the inhibitory factors are higher in seasonal-flowering orchids. A considerable proportion of our transcriptome data included genes that function in hormone signaling. We identified auxin homeostatic and signaling gene families, including ARFs, GH3, IAAs, PINs, SAURs and YUCs; PINs were expressed specifically at the early stages of floral development. We also detected several genes related to gibberellins in the first two stages of flower development (Supplementary Fig. 5). The expression of gibberellin biosynthesis genes (GA3OX and GA20OX) and GA signaling DELLA genes (GAI and SLR1) was high in the early stages of flower development (Fig. 5). Transcriptomic analysis shows elevated expression of FT/FD and GA biosynthesis-related genes, which promote growth, and the downregulation of ABA pathway genes at the same time may transiently release dormancy to ease bud break [57-59]. However, ABA may defy this effect in two ways: it may downregulate FT/FD at low temperatures and regulate GA levels by inhibiting SVP during short-days.

SVP (SHORT VEGETATIVE PHASE) is a floweringtime regulator. It positively regulates TCP18 [TEOSINTE BRANCHED, CYCLOIDEA, PCR (TCP)], which is a mediator of temperature-dependent bud break [60]. SVP and TCP function in a temperature-sensitive transcriptional module and mediate bud break. Moreover, SVP targets the ABA and GA pathway genes during bud break [61]. SVP also mediates photoperiodic control of dormancy, downstream of the ABA pathway, in hybrid aspen. Downregulation of SVP suppresses dormancy, whereas overexpression alleviates the dormancy defects caused by ABA insensitivity [60]. We identified two homologs of Arabidopsis thaliana SVP, showing the highest expression in leaves (Fig. 5a). Among the TCP homologs identified, TCP3 showed the highest expression in FD1-FD3 and TCP21 was expressed in FD1 and leaves.

Taken together, we present that multiple regulatory pathways may control flowering regulation in A. graminifolia (Fig. 5c). We found candidate genes from multiple regulatory conduits: photoperiod, circadian clock, vernalization, hormonal, and autonomous pathways. These 
pathways interact with floral integrators to regulate flowering time. FT1/FD may act as a central receiver of signals along with APETALA (AP1, AP2 and AP3) and SOC1. Moreover, ABA may control bud break through SVP at low temperature during short days. However, extended research is required to reveal these hypotheses in A. graminifolia.

\section{Conclusions}

Our results show that different pathways coordinate the early and late stages of flower development. We found key regulatory genes of flowering time for different pathways, such as photoperiod (EFL3, COLs), vernalization (VIL2), circadian clock (MYB108 and GI), and autonomous (FCA, FPA, FY) pathways, and the key floral integrators of these pathways (FT, SEP, FRI, TCP3, AGL5, MADS6, and MADS16). In the hormonal pathway, we found key regulators of gibberellin (GA20OX, GA3OX, GAI, and SLR1) and abscisic acid (PYL4, ABF1, ABF2, and CYP707A5). All these genes were validated through qRT-PCR (Fig. 5). Major plant hormones were quantified in A. graminifolia and compared with typical seasonal flowering orchid species (C. sinense and C. ensifolium). Therefore, this mining of key regulators from different pathways sets a basis for functional characterization of these genes in orchids to decipher the complete model of flowering time regulation.

\section{Methods}

Plant materials and growth conditions

Plants of Arundina graminifolia were grown in three groups from seeds at the greenhouse of the Environmental Horticultural Research Institute of Guangdong Academy of Agricultural Sciences, China. All the groups were maintained at a day/night temperature of $25 / 20^{\circ} \mathrm{C}$ and photoperiod of $16 / 8 \mathrm{~h}$. Sampling was done from five floral developmental (FD) stages (stage 1-5), mature flowers, leaves, root and capsule. Nine samples were obtained for each stage of flower development or tissue type from each group. By this, each tissue was sampled 27 times from three groups. Samples were pooled and three samples were collected for RNA-sequencing, while the remaining samples were used for hormonal analyses. Thus, three technical and biological repeats were collected from each stage and tissue type. Samples were collected in liquid nitrogen and immediately stored at $-80^{\circ} \mathrm{C}$ until the extraction of RNA.

\section{RNA-seq library preparation and sequencing}

RNA was extracted from various tissue types (5 floral developmental stages, mature flower, leaf, root and capsule) using TaKaRa kit for RNA extraction. The cDNA libraries were constructed from RNA. The mRNAs were filtered from total RNA using Oligotex mRNA Midi Kit (QIAGEN, GERMANY). RNA was quantified on NanoDrop 2000 spectrophotometer (Thermo Scientific, USA) and the cDNA libraries were generated following Illumina manufacturing protocol as suggested previously [62]. In short, mRNAs were obtained from total RNA and fragments were made to an approximate length of $200 \mathrm{bp}$. Then, the isolated mRNAs were subjected to cDNA synthesis of first and second strand followed by adapter ligation and low-cycle enrichment following the instructions by TruSeq ${ }^{\circledR}$ RNA HT Sample Prep Kit (Illumina, USA). The library products thus purified were evaluated using the Agilent 2200 TapeStation and Qubit ${ }^{\circledR} 2.0$ (Life Technologies, USA), followed by dilution to $10 \mathrm{pM}$ for in situ cluster generation on the HiSeq2500 pair-end flow cell and then pair end sequencing $(2 \times 100 \mathrm{bp})$ was performed. About 60 million reads were produced for each sample. Transcriptomic de novo assembly was performed using Trinity program with default parameters [63].

\section{DEG analysis}

The level of gene expression was ascertained by RPKM value using the following formula:

$\mathrm{RPKM}=[$ total exon reads/mapped reads (millions) $] \mathrm{x}$ exon length $(\mathrm{kb})$

The significant difference of gene expression among various tissue types was determined using edgeR. The false discovery rate (FDR) was used to determine the threshold $P$-value in various tests and a threshold level of significant difference in gene expression was set at FDR $<0.05$ and | $\log 2$ ratio $\mid>1$ (two fold change). Pearson correlation coefficient (PCC) was used to ascertain the correlation between different parts. Moreover, principal component analysis (PCA) and hierarchical clustering were performed using corrplot and prcomp utilities of R package [64].

The DEGs were annotated to Kyoto Encyclopedia of Genes and Genomes (KEGG) and gene ontology (GO) enrichment analyses following the method described previously [65]. Briefly, all the DEGs were first mapped to GO terms or KEGG pathways in the databases (http:// www.geneontology.org/ or http://www.genome.ad.jp/), calculating gene number for each term or pathway [66, 67]. Then, the hypergeometric test was applied to find the DEGs with genetically enriched terms. A threshold $p$-value or $q$-value of $\leq 0.05$ was set to filter significantly enriched GO terms or KEGG pathways.

\section{GO and pathway enrichment analyses}

Clustering of selective DEGs was performed for biological process using BINGO plug-in of Cytoscape [64, 68]. For GO terms, the $P$-value was corrected following the method by Benjamini Hoschberg [64]. The GO terms with $q$-value $\leq 0.05$ were considered as significantly 
enriched. Pathway enrichment analysis of different gene sets was performed using MapMan (v3.6.0RC1) with the best Arabidopsis (TAIR10) homolog as reference.

\section{Identification of Transcription Factor (TF) families}

The term 'transcription factor' was searched across annotated DEGs, and identified 687 DEGs annotated as transcription factors. The TFs were sorted into $35 \mathrm{TF}$ families.

\section{Weighted Gene Coexpression Network Analysis (WGCNA)}

WGCNA was performed to identify modules related to flowering using the WGCNA packages in $\mathrm{R}$, as previously reported [69].

\section{Real-time quantitative RT-PCR analysis}

A total of 32 genes (30 hormonal and flowering pathway genes, and 2 FT homologs) were selected to check their expression trends through qRT-PCR across different plant positions. The RNA was extracted from 27 samples of 9 tissues of the plants grown in the green house of the academy as mentioned above. Total RNA was extracted from eight tissue types and reverse-transcribed to obtain cDNA following protocol by Fermentas. The cDNA was subjected to qRT-PCR in a reaction mixture volume of $20 \mu \mathrm{l}$ containing $10 \mu \mathrm{l}$ of SYBR premix Ex-taq ${ }^{\mathrm{TM}}$ (Takara, Japan) using Bio-Rad CFX-96 RealTime PCR System (Bio-Rad, USA). Actin was used as an internal standard for normalization of expression data. The experiment was performed in three biological replicates, each containing three technical replicates. The primers to clone the targeted genes were designed using Primer 5.0 software (Supplementary Table 3).

\section{Hormonal analysis}

Hormonal analysis was performed from five stages of floral development to compare hormonal contents for Cymbidium sinense, Cymbidium ensifolium and Arundina graminifolia. C. sinense and C. ensifolium are typical seasonal flowering species and therefore $A$. graminifolia was compared with them to make a comparison of hormonal fluctuations between season and continuous flowering. Hormonal contents were determined following the protocol of HPLC-MS/MS (Aglient) described by Pan et al. [70].

\section{Scanning electron microscopy}

Floral buds were collected from stage 0 to stage 2 for microscopic detailing of floral initiation. Buds were dissected and fixed in a solution containing $2 \%$ formaldehyde and $3 \%$ glutaraldehyde for $24 \mathrm{~h}$. After that dehydration was done in acetone and dried at a criticalpoint in liquid $\mathrm{CO}_{2}$. Fully dried samples were fixed on stubs and sputter bearing $25 \mathrm{~nm}$ coating of gold. Finally, the specimens were observed under a scanning electron microscope JSM-6360LV (JEOL).

\section{Statistical analysis}

One-way ANOVA was applied using SPSS software (SPSS Inc., Chicago, IL, USA; ver. 16.0) to analyse the hormonal and qRT-PCR data. Significant differences are shown at $p<0.05$ or $p<0.01$ level.

\section{Supplementary Information}

The online version contains supplementary material available at https://doi. org/10.1186/s12870-021-03350-6.

Additional file 1: Supplementary Figure 1 Plants of A. graminifolia A: Whole plant; B: Opening flower; C: Mature flower. Supplementary Figure 2. Annotation overview. A: eggNOG functional categories; B: GO categories; C: KEGG pathway classification; D: Shared and unique proteins among A. graminifolia, Apostasia shenzhenica, Dendrobium candidum, and Phalaenopsis equestris. Supplementary Figure 3. Abundance of TF families; a) number of TFs in all major families, b) bHLH TF family, c) MYB TF family, d) WRKY TF family. Supplementary Figure 4. a) Number of up and down regulated TFs, b) stage specific number of up and down regulated TFs, c) relation of up and down regulation across different tissues. Supplementary Figure 5. Search results of annotation of "Gibberellins" throughout the DEGs.

Additional file 2: Supplementary Table 1. Flower development and plant position-based division of transcriptomic data. Supplementary Table 2. Total number of transcripts and unigenes identified in the transcriptome. Supplementary Table 3. Primers used for qRT-PCR. Supplementary Table 4. Similarity index of some of the important flowering related DEGs in Arundina graminifolia with other orchids and angiosperms.

\section{Acknowledgements}

We are thankful to funding agencies for funding and Plant Editors for providing professional services for language editing and final polishing of our manuscript.

\section{Code availability}

Not applicable.

\section{Authors' contributions}

Sagheer Ahmad: Conceptualization, Software, Writing-original draft; Chuqiao Lu: Data curation, Formal analysis; Jie Gao: Data curation, Formal analysis, Investigation; Rui Ren: Visualization, Investigation; Yonglu Wei: Visualization, Investigation; Jieqiu Wu: Data curation, Formal analysis; Jianpeng Jin: Software, Validation; Chuanyuan Zheng: Data curation, Formal analysis; Genfa Zhu: Supervision, Conceptualization, Funding acquisition; Fengxi Yang: Supervision, Conceptualization, Funding acquisition, Writing-Reviewing and editing. The author(s) read and approved the final manuscript.

\section{Funding}

This research was funded by the grants from R\&D plan in key areas of Guangdong Province (2018B020202001), Natural Science Foundation of Guangdong Province (2017A030312004), Guangdong modern agricultural industrial technology system flower innovation team expert project (2021 KJ121), Guangzhou scientific research project (201904020026), and the Guangdong Academy of Agricultural Sciences Discipline Team Construction Project (202127 TD, BZ202006, R2020PY-JX018).

\section{Availability of data and materials}

All relevant supplementary data is provided along with this manuscript as supplementary file. 


\section{Declarations}

Ethics approval and consent to participate

Not applicable.

\section{Consent for publication}

Not applicable.

\section{Competing interests}

The authors declare that they have no competing interest.

Received: 26 August 2021 Accepted: 17 November 2021

Published online: 10 December 2021

\section{References}

1. Han R, Truco MJ, Lavelle DO, Michelmore RW. A Composite Analysis of Flowering Time Regulation in Lettuce. Front Plant Sci. 2021;12:632708.

2. Zhang J, Wu K, Zeng S, Teixeira da Silva JA, Zhao X, Tian CE, et al. Transcriptome analysis of Cymbidium sinense and its application to the identification of genes associated with floral development. BMC Genomics. 2013;14:279.

3. Teotia S, Tang G. To bloom or not to bloom: role of microRNAs in plant flowering. Mol Plant. 2015;8(3):359-77.

4. Komeda Y. Genetic regulation of time to flower in Arabidopsis thaliana. Annu Rev Plant Biol. 2004;55:521-35.

5. Aceto S, Gaudio L. The MADS and the beauty: genes involved in the development of orchid flowers. Curr Genomics. 2011;12(5):342-56.

6. Yang F, Zhu G, Wang Z, Liu H, Xu Q, Zhao C. Integrated mRNA and microRNA transcriptome variations in the multi-tepal mutant provide insights into the floral patterning of the orchid Cymbidium goeringii. BMC Genomics. 2017;18(1):367.

7. Hou CJ, Yang CH. Functional analysis of FT and TFL1 orthologs from orchid (Oncidium Gower Ramsey) that regulate the vegetative to reproductive transition. Plant Cell Physiol. 2009;50(8):1544-57.

8. Liu XR, Pan T, Liang WQ, Gao L, Wang XJ, Li HQ, et al. Overexpression of an orchid (Dendrobium nobile) SOC1/TM3-like Ortholog, DnAGL19, in Arabidopsis regulates HOS1-FT expression. Front Plant Sci. 2016;7:99.

9. Jang S. Functional characterization of PhapLEAFY, a FLORICAULA/ LEAFY Ortholog in Phalaenopsis aphrodite. Plant Cell Physiol. 2015;56(11):2234-47.

10. Lin YF, Chen YY, Hsiao YY, Shen CY, Hsu JL, Yeh CM, et al. Genome-wide identification and characterization of TCP genes involved in ovule development of Phalaenopsis equestris. J Exp Bot. 2016;67(17):5051-66.

11. Lin CS, Chen JJ, Huang YT, Hsu CT, Lu HC, Chou ML, et al. Catalog of Erycina pusilla miRNA and categorization of reproductive phase-related miRNAs and their target gene families. Plant Mol Biol. 2013;82(1-2):193-204.

12. Chou ML, Shih MC, Chan MT, Liao SY, Hsu CT, Haung YT, et al. Global transcriptome analysis and identification of a CONSTANS-like gene family in the orchid Erycina pusilla. Planta. 2013;237(6):1425-41.

13. Jang S, Choi S-C, Li H-Y, An G, Schmelzer E. Functional characterization of Phalaenopsis aphrodite flowering genes PaFT1 and PaFD. PLoS One. 2015;10(8):e0134987.

14. Park SJ, Jiang K, Tal L, Yichie Y, Gar O, Zamir D, et al. Optimization of crop productivity in tomato using induced mutations in the florigen pathway. Nat Genet. 2014;46(12):1337-42

15. Moraes TS, Dornelas MC, Martinelli AP. FT/TFL1: Calibrating Plant Architecture. Front Plant Sci. 2019;10:97.

16. Wang S-L, Viswanath KK, Tong C-G, An HR, Jang S, Chen F-C. Floral Induction and Flower Development of Orchids. Front Plant Sci. 2019:10:1258.

17. Goh C, Yang A. Effects of growth regulators and decapitation on flowering of Dendrobium orchid hybrids. Plant Sci Lett. 1978;12(3-4):287-92.

18. Bhalerao RP, Bennett MJ. The case for morphogens in plants. Nat Cell Biol. 2003;5(11):939-43.

19. Benková E, Ivanchenko MG, Friml J, Shishkova S, Dubrovsky JG. A morphogenetic trigger: is there an emerging concept in plant developmental biology? Trends Plant Sci. 2009;14(4):189-93.

20. Möller B, Weijers D. Auxin control of embryo patterning. Cold Spring Harb Perspect Biol. 2009;1(5):a001545.
21. Lau S, De Smet I, Kolb M, Meinhardt H, Jürgens $G$. Auxin triggers a genetic switch. Nat Cell Biol. 2011;13(5):611-5.

22. Finet $C$, Jaillais $Y$. Auxology: when auxin meets plant evo-devo. Dev Biol. 2012;369(1):19-31.

23. Zoulias N, Duttke SHC, Garcês H, Spencer V, Kim M. The role of Auxin in the pattern formation of the Asteraceae flower head (Capitulum). Plant Physiol. 2019;179(2):391-401.

24. Hew C, Clifford P. Plant growth regulators and the orchid cut-flower industry. Plant Growth Regul. 1993:13(3):231-9.

25. Ding L, Wang Y, Yu H. Overexpression of DOSOC1, an ortholog of Arabidopsis SOC1, promotes flowering in the orchid Dendrobium Chao Parya smile. Plant Cell Physiol. 2013;54(4):595-608.

26. Hyun Y, Richter R, Vincent C, Martinez-Gallegos R, Porri A, Coupland G. Multi-layered regulation of SPL15 and cooperation with SOC1 integrate endogenous flowering pathways at the Arabidopsis shoot meristem. Dev Cell. 2016:37(3):254-66.

27. Jan A, Kitano H, Matsumoto $H$, Komatsu S. The rice OsGAE1 is a novel gibberellin-regulated gene and involved in rice growth. Plant Mol Biol. 2006;62(3):439-52.

28. Iwamoto M, Kiyota S, Hanada A, Yamaguchi S, Takano M. The multiple contributions of phytochromes to the control of internode elongation in rice. Plant Physiol. 2011;157(3):1187-95.

29. Li J, Jiang J, Qian Q, Xu Y, Zhang C, Xiao J, et al. Mutation of rice BC12/ GDD1, which encodes a kinesin-like protein that binds to a GA biosynthesis gene promoter, leads to dwarfism with impaired cell elongation. Plant Cell. 2011;23(2):628-40.

30. Wang $Y, L i ~ L, ~ Y e T, L u Y, C h e n X, W u Y$. The inhibitory effect of $A B A$ on floral transition is mediated by ABI5 in Arabidopsis. J Exp Bot. 2013;64(2):675-84.

31. Shu K, Chen Q, Wu Y, Liu R, Zhang H, Wang S, et al. ABSCISIC ACIDINSENSITIVE 4 negatively regulates flowering through directly promoting Arabidopsis FLOWERING LOCUS C transcription. J Exp Bot. 2016;67(1):195-205.

32. Bouwmeester HJ, Matusova R, Zhongkui S, Beale MH. Secondary metabolite signalling in host-parasitic plant interactions. Curr Opin Plant Biol. 2003:6(4):358-64.

33. Hayward A, Stirnberg P, Beveridge C, Leyser O. Interactions between auxin and strigolactone in shoot branching control. Plant Physiol. 2009;151(1):400-12.

34. Alder A, Jamil M, Marzorati M, Bruno M, Vermathen $M$, Bigler $P$, et al. The path from $\beta$-carotene to carlactone, a strigolactone-like plant hormone Science. 2012;335(6074):1348-51.

35. Cai J, Liu X, Vanneste K, Proost S, Tsai W-C, Liu K-W, et al. The genome sequence of the orchid Phalaenopsis equestris. Nat Genet. 2015;47(1):65.

36. Wong DC, Pichersky E, Peakall R. The biosynthesis of unusual floral volatiles and blends involved in orchid pollination by deception: current progress and future prospects. Front Plant Sci. 2017;8:1955.

37. Yang F, Zhu G, Wei Y, Gao J, Liang G, Peng L, et al. Low-temperatureinduced changes in the transcriptome reveal a major role of CgSVP genes in regulating flowering of Cymbidium goeringii. BMC Genomics. 2019;20(1):53.

38. Seidenfaden G, Wood JJ, Holttum RE. The orchids of peninsular Malaysia and Singapore: Olsen \& Olsen; 1992.

39. Hooker J. Gnetaceae. Flora Br India. 1890:5:640-3.

40. Auberon F, Olatunji OJ, Krisa S, Antheaume C, Herbette G, Bonté F, et al. Two new stilbenoids from the aerial parts of Arundina graminifolia (Orchidaceae). Molecules. 2016;21(11):1430.

41. Wu ZY, Raven PH, Hong DY. Flora of China. Volume 25 (Orchidaceae). Beijing: Science Press and Missouri Botanical Garden Press; 2009.

42. Ai Y, Xie T-X, Liu D-K, Tu X-D, Zhou J, Liu Z-J. Complete chloroplast genome of Arundina graminifolia (Orchidaceae). Mitochondrial DNA Part B. 2019;4(2):2898-9.

43. Abe M, Kobayashi Y, Yamamoto S, Daimon Y, Yamaguchi A, Ikeda Y, et al. FD, a bZIP protein mediating signals from the floral pathway integrator FT at the shoot apex. Science. 2005:309(5737):1052-6.

44. Wigge PA, Kim MC, Jaeger KE, Busch W, Schmid M, Lohmann JU, et al. Integration of spatial and temporal information during floral induction in Arabidopsis. Science. 2005;309(5737):1056-9.

45. Corbesier L, Vincent C, Jang S, Fornara F, Fan Q, Searle I, et al. FT protein movement contributes to long-distance signaling in floral induction of Arabidopsis. Science. 2007;316(5827):1030-3. 
46. Mandaokar A, Browse J. MYB108 acts together with MYB24 to regulate jasmonate-mediated stamen maturation in Arabidopsis. Plant Physiol. 2009;149(2):851-62.

47. Ono S, Liu H, Tsuda K, Fukai E, Tanaka K, Sasaki T, et al. EAT1 transcription factor, a non-cell-autonomous regulator of pollen production, activates meiotic small RNA biogenesis in rice anther tapetum. PLoS Genet. 2018;14(2):e1007238.

48. Chandler J, Werr W. A phylogenetically conserved APETALA2/ethylene response factor, ERF12, regulates arabidopsis floral development. Plant Mol Biol. 2020;102(1):39-54.

49. Yant L, Mathieu J, Dinh TT, Ott F, Lanz C, Wollmann H, et al. Orchestration of the floral transition and floral development in Arabidopsis by the bifunctional transcription factor APETALA2. Plant Cell. 2010;22(7):2156-70

50. Halliday KJ, Salter MG, Thingnaes E, Whitelam GC. Phytochrome control of flowering is temperature sensitive and correlates with expression of the floral integrator FT. Plant J. 2003;33(5):875-85.

51. Barbier FF, Lunn JE, Beveridge CA. Ready, steady, go! A sugar hit starts the race to shoot branching. Curr Opin Plant Biol. 2015;25:39-45.

52. Beveridge CA, Dun EA, Rameau C. Pea has its tendrils in branching discoveries spanning a century from auxin to strigolactones. Plant Physiol. 2009;151(3):985-90.

53. Müller D, Leyser O. Auxin, cytokinin and the control of shoot branching. Ann Bot. 2011;107(7):1203-12.

54. Kebrom TH, Spielmeyer W, Finnegan EJ. Grasses provide new insights into regulation of shoot branching. Trends Plant Sci. 2013;18(1):41-8.

55. Wang $\mathrm{H}$, Wang $\mathrm{H}$. Phytochrome signaling: time to tighten up the loose ends. Mol Plant. 2015;8(4):540-51.

56. Yuan C, Xi L, Kou Y, Zhao Y, Zhao L. Current perspectives on shoot branching regulation. Front Agric Sci Eng. 2015;2(1):38-52.

57. Horvath DP, Chao WS, Suttle JC, Thimmapuram J, Anderson JV. Transcriptome analysis identifies novel responses and potential regulatory genes involved in seasonal dormancy transitions of leafy spurge (Euphorbia esula L.). BMC Genomics. 2008;9:536.

58. Bai S, Saito T, Sakamoto D, Ito A, Fujii H, Moriguchi T. Transcriptome analysis of Japanese pear (Pyrus pyrifolia Nakai) flower buds transitioning through endodormancy. Plant Cell Physiol. 2013;54(7):1132-51.

59. Rinne PL, Welling A, Vahala J, Ripel L, Ruonala R, Kangasjärvi J, van der Schoot C. Chilling of dormant buds hyperinduces FLOWERING LOCUST and recruits GA-inducible 1,3-beta-glucanases to reopen signal conduits and release dormancy in Populus. Plant Cell. 2011;23(1):130-46. https:// doi.org/10.1105/tpc.110.081307. Epub 2011 Jan 31.PMID: 21282527; PMCID: PMC3051240.

60. Singh RK, Miskolczi P, Maurya JP, Bhalerao RP. A tree ortholog of SHORT VEGETATIVE PHASE floral repressor mediates photoperiodic control of bud dormancy. Curr Biol. 2019;29(1):128-33 e122.

61. Singh RK, Maurya JP, Azeez A, Miskolczi P, Tylewicz S, Stojkovič K, et al. A genetic network mediating the control of bud break in hybrid aspen. Nat Commun. 2018;9(1):4173.

62. Kang YJ, Kim SK, Kim MY, Lestari P, Kim KH, Ha BK. Genome sequence of mungbean and insights into evolution within Vigna species. Nat Commun. 2014;5:5443.

63. Grabherr MG, Haas BJ, Yassour M, Levin JZ, Thompson DA, Amit I, et al. Full-length transcriptome assembly from RNA-Seq data without a reference genome. Nat Biotechnol. 2011;29(7):644.

64. Garg R, Singh VK, Rajkumar MS, Kumar V, Jain M. Global transcriptome and coexpression network analyses reveal cultivar-specific molecular signatures associated with seed development and seed size/weight determination in chickpea. Plant J. 2017;91(6):1088-107.

65. Wang Y, Huang H, Ma YP, Fu JX, Wang LL, Dai SL. Construction and de novo characterization of a transcriptome of Chrysanthemum lavandulifolium: analysis of gene expression patterns in floral bud emergence. Plant Cell Tiss Org. 2014;116(3):297-309.

66. Conesa A, Gotz S, Garcia-Gomez JM, Terol J, Talon M, Robles M. Blast2GO: a universal tool for annotation, visualization and analysis in functional genomics research. Bioinformatics. 2005:21(18):3674-6.

67. Kanehisa M, Goto S. KEGG: Kyoto encyclopedia of genes and genomes. Nucleic Acids Res. 2000;28(1):27-30

68. Maere S, Heymans K, Kuiper M. BiNGO: a Cytoscape plugin to assess overrepresentation of gene ontology categories in biological networks. Bioinformatics. 2005:21(16):3448-9.
69. Ahmad S, Yuan C, Yang Q, Yang Y, Cheng T, Wang J, et al. Morpho-physiological integrators, transcriptome and coexpression network analyses signify the novel molecular signatures associated with axillary bud in chrysanthemum. BMC Plant Biol. 2020;20:1-15.

70. Pan X, Welti R, Wang X. Quantitative analysis of major plant hormones in crude plant extracts by high-performance liquid chromatography-mass spectrometry. Nat Protoc. 2010;5(6):986.

\section{Publisher's Note}

Springer Nature remains neutral with regard to jurisdictional claims in published maps and institutional affiliations.
Ready to submit your research? Choose BMC and benefit from:

- fast, convenient online submission

- thorough peer review by experienced researchers in your field

- rapid publication on acceptance

- support for research data, including large and complex data types

- gold Open Access which fosters wider collaboration and increased citations

- maximum visibility for your research: over $100 \mathrm{M}$ website views per year

At BMC, research is always in progress.

Learn more biomedcentral.com/submissions 